\title{
Developing targets for radiation transport experiments at the Omega laser facility
}

\author{
D. Capelli, C.A. Charsley-Groffman, R.B. Randolph, D.W. Schmidt, T. Cardenas, F. Fierro, G. Rivera, \\ C. Hamilton, J.D. Hager, H. M. Johns, N. E. Lanier, and J.L. Kline \\ Los Alamos National Laboratory, Los Alamos, NM, USA \\ (Received 5 December 2016; revised 17 March 2017; accepted 5 May 2017)
}

\begin{abstract}
Targets have been developed to measure supersonic radiation transport in aerogel foams using absorption spectroscopy. The target consists of an aerogel foam uniformly doped with either titanium or scandium inserted into an undoped aerogel foam package. This creates a localized doped foam region to provide spatial resolution for the measurement. Development and characterization of the foams is a key challenge in addition to machining and assembling the two foams so they mate without gaps. The foam package is inserted into a beryllium sleeve and mounted on a gold hohlraum. The target is mounted to a holder created using additive manufacturing and mounted on a stalk. The manufacturing of the components, along with assembly and metrology of the target are described here.
\end{abstract}

Keywords: absorption spectroscopy; doped foams; radiation transport

\section{Introduction}

When a supersonic radiation wave passes through a material, it heats the material on time scales much faster than the bulk motion of the material which is much closer to the material sound speed. Since there is no motion of the material behind the heat front, there is little change in density. Thus, conventional $\mathrm{x}$-ray radiography cannot be used to track the position of the heat front. One way to track the supersonic heat front is to measure the spatial distribution of the material temperature using imaging absorption spectroscopy. For absorption spectroscopy, a tracer element is needed to produce an absorption spectrum. In previous experiments, chlorinated foams were used where the chlorine serves as the tracer ${ }^{[1-3]}$. However, because the ionization state for chlorine is very stable above $\sim 100 \mathrm{eV}$, the absorption spectra it generates are insensitive to changes in temperature. Experiments on the National Ignition Facility (NIF) ${ }^{[4]}$ where the material temperature reaches closer to $\sim 200 \mathrm{eV}$ require a higher- $z$ tracer element. To address this issue, targets using aerogel foams ${ }^{[5]}$ doped with fine particles of titanium and scandium have been developed and are being tested ${ }^{[6]}$ at the Omega laser facility ${ }^{[7]}$. Aerogel foams can be doped uniformly with these materials, as well as in small clusters for radiation transport experiments. A key development for these targets is the ability to provide spatial resolution in

Correspondence to: D. Capelli, Los Alamos National Laboratory, MS E519, Los Alamos, NM, 87544, USA. Email: dcapelli@lanl.gov. the transverse direction to the heat wave (the non-imaging direction) by having only a region of the foam package doped. Figure 1 shows an $\mathrm{Xradia}^{[8]}$ radiograph of a typical COAX target. For these targets, a cylinder of doped aerogel foam is inserted into a larger undoped aerogel foam. The foam is placed in a beryllium sleeve and mounted to the top of a gold hohlraum. Details of the manufacturing, assembly and characterization of these targets are presented.

\section{Design}

The design for the COAX targets is shown in Figure 2. Figure 2(a) shows the foam package design with the doped aerogel foam region signified in gray hash. The tolerance of the doped foam is +0.0 and $-5.0 \mu \mathrm{m}$ to ensure the foam is smaller than the specification. Similarly, the inner dimensions of the undoped foam are between +0.0 and $-5.0 \mu \mathrm{m}$, smaller as well. The tolerance of the foams is the most important specification, set so the foams are not larger than the specified size to ensure the foams can be assembled without damage and the foam stick together without glue. The tight tolerance keeps the gap between the two foams small enough to ensure the radiation is transported through the foams and not along the gap. The foam density can be varied between $\sim 50$ and $80 \mathrm{mg} / \mathrm{mL}$ with $\sim 1: 5$ ratio of dopant material to aerogel. Machinability of the foam sets the lower density limit while laser energy sets the upper density limit. Foam with both $\mathrm{Ti}$ and Sc dopants have been 


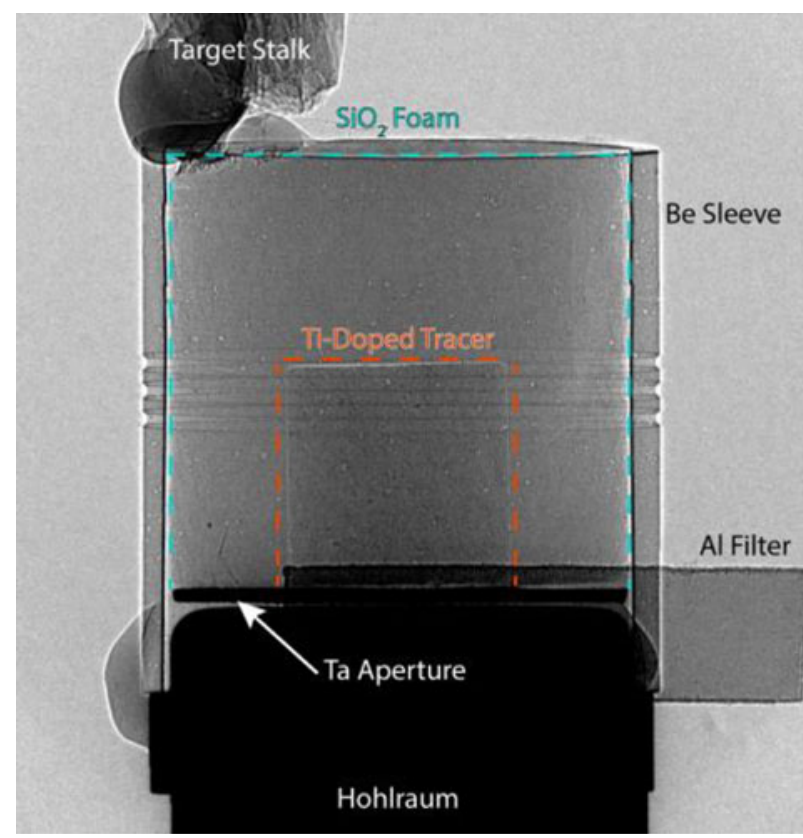

Figure 1. X ray radiograph of COAX target using the Xradia.

manufactured. The surrounding foam is undoped aerogel foam usually with a slightly higher density than the doped foam between 50 and $80 \mathrm{mg} / \mathrm{mL}$ to maintain similar electron densities and temperatures between the two foam regions. Figure 2(b) shows the drawing of the assembled target with the hohlraum and the foam package. To increase robustness, the foam package is mounted in a beryllium tube that is attached to the hohlraum. Figure 2(c) shows the final target assembly including the stalk. Another feature is a collar on the outside of the hohlraum to support the beryllium tube for easy and reproducible assembly of the target. The hohlraums are manufactured by General Atomics ${ }^{[9]}$.

On a historical note, the target design has evolved. The original design of these targets deposited near solid density $\mathrm{Ti}$ on one half of the foam that had been split into two parts ${ }^{[10]}$. The foams were reunited and mounted. However, the dense titanium absorbs more x-rays from the hohlraum than the surrounding foam leading to a pressure imbalance not acceptable for the experiments. The second iteration used a rectangular block of doped foam with a slot in the undoped foam to simplify machining (Figure 3). While this configuration succeeded in providing absorption spectra, the orthogonal views of the two instruments measuring spectra and subsonic foam motion are not the same. In addition, it is more difficult to keep the slotted foam in the undoped foam while preventing glue from wicking into the experimental region of the foam package. The current design is the third iteration. The beryllium tube has been added to make the assembly more robust. The initial designs mounted the foam directly to the aperture and on occasion, the foams would get damaged, separate from the hohlraum or glue would wick into the foam rendering the target unacceptable for a)
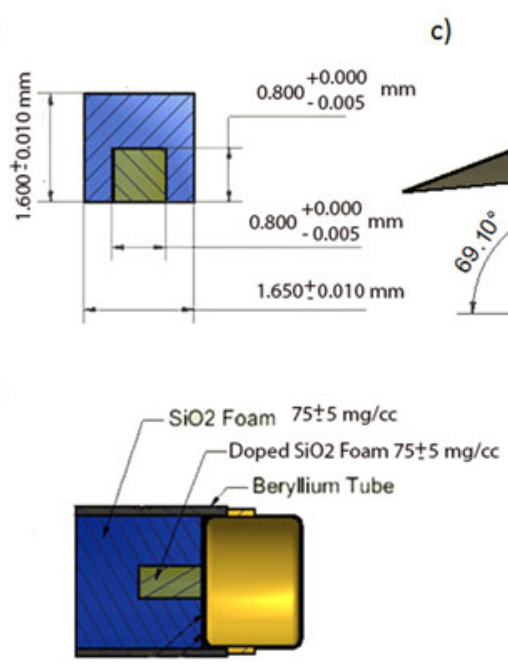

b)

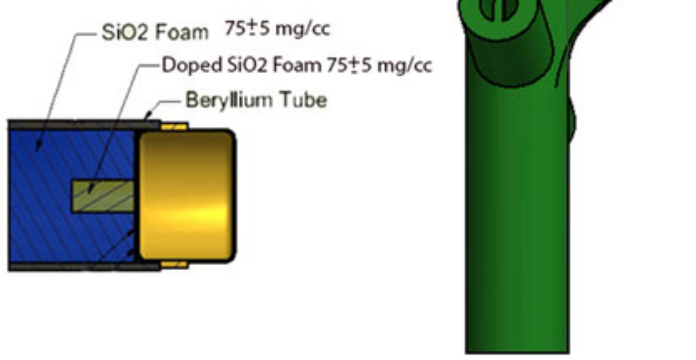

Figure 2. (a) Foam target designs with aperture, (b) hohlraum foam assembly, (c) final target assembly with stalk mounting.
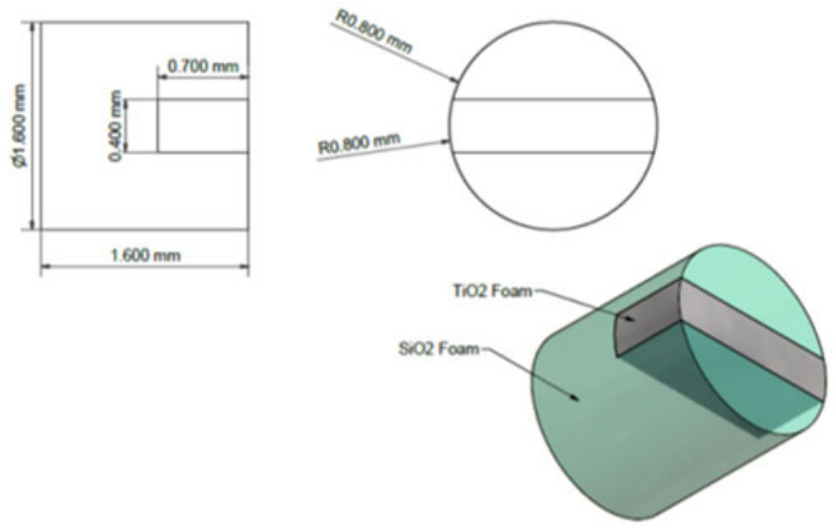

Figure 3. Schematic of foam with slot insert.

the experiments. The beryllium sleeve protects the foam and holds it in place and a larger amount of glue can be used between the hohlraum and beryllium sleeve away from the experimental region. The beryllium sleeve was never used for the slotted foam design, but would have prevented glue from spreading into the experimental line of sight, thus making the target more robust.

\section{Ti- and Sc-doped foams}

Creating the doped aerogel foams with high- $z$ particles uses previously developed technology ${ }^{[11]}$. All chemicals are off the shelf with no further purification required. The solution starts with $12 \mathrm{~g}$ of methyl silicate 51 precursor 
from BOC sciences ${ }^{[12]}$ (50\% solids by mass) yielding $6 \mathrm{~g}$ (0.1 mol). This is diluted to $100 \mathrm{~mL}$ with methanol (Fisher Scientific $\left.{ }^{[13]}\right) .1 .6 \mathrm{~g}$ of titania $\left(\mathrm{TiO}_{2}\right)$ nanoparticle powder from Sigma-Aldrich ${ }^{[14]}$ (22 $\mathrm{nm}$ nominal particle size) is added containing $0.02 \mathrm{~mol} \mathrm{TiO}_{2}$. Finally, $3 \mathrm{~mL}$ of $5 \mathrm{~mol} / \mathrm{L}$ aqueous ammonium hydroxide (Fisher Scientific) is added to catalyze gelation (typical gel times were 20-30 min). The mixture is placed in a bath sonicator and mixed until viscosity begins to increase enough to prevent $\mathrm{TiO}_{2}$ from sedimenting. The mixture is quickly sealed in stainless steel molds (before reaching the gel point) which are aged $24 \mathrm{~h}$ at room temperature before supercritical drying.

Supercritical drying is achieved by placing the molds in a methanol filled autoclave. The system is pressurized to $140 \mathrm{~atm}$ and heated to $300^{\circ} \mathrm{C}$ over $4 \mathrm{~h}$, maintaining constant pressure by slowly releasing methanol. The vessel is then held at constant temperature and pressure (140 atm, $300^{\circ} \mathrm{C}$ ) for $1 \mathrm{~h}$. Pressure is slowly released over $2 \mathrm{~h}$ while maintaining temperature. Finally, the reactor is placed under vacuum and allowed to cool to room temperature overnight. Producing the desired foam density is challenging due to volumetric shrinkage that can increase the density by $15 \%-$ $20 \%$.

$\mathrm{Sc}$-doped $\mathrm{SiO}_{2}$ is prepared in exactly the same way except that the amount of methanol used is $120 \mathrm{~mL}$ and $2.76 \mathrm{~g}$ of $\mathrm{Sc}_{2} \mathrm{O}_{3}$ powder $(7 \mu \mathrm{m}$ nominal particle size) from Goodfellow inc. ${ }^{[15]}$. $0.02 \mathrm{~mol}$ is added to the $12 \mathrm{~g}$ of methyl silicate. Ideally, the Sc particles would be much smaller $<0.5 \mu \mathrm{m}$. Efforts to reduce their size via mortar and pestle did not work. Currently, an effort is underway to use Sc nanoparticles to form more uniform distribution of $\mathrm{Sc}$ in the foam. This will allow the Sc dopant to ionize and to equilibrate with the surrounding foam faster.

Once the foams are completed, the foams are characterized using both gravimetric measurements and $\mathrm{x}$-ray transmission. First, the difference in mass between the pure $\mathrm{SiO}_{2}$ bulk sample (30 mm long $\times 2 \mathrm{~mm}$ diameter cylinders) and the Ti-doped $\mathrm{SiO}_{2}$ bulk sample is used to infer the relative concentration of $\mathrm{Ti}$ in the doped sample. The machined Tidoped foams are not massive enough to measure the density gravimetrically. After the individual samples are machined the density is characterized again using the density characterization station $(\mathrm{DCS})^{[16]}$, a mono-energetic chromium $\mathrm{x}$ ray imaging system at $5.6 \mathrm{keV}$. A CCD camera images the $\mathrm{x}$-rays transmitted through the samples as shown in Figure 4. From the path length through the foam, the transmission and cold opacity, the density can be determined. The cold opacity is derived from the Henke tables based on the foam stoichiometry. The largest uncertainty in the density measurement is the stoichiometry. The uniformity of the doped and the undoped foams is also determined from the DCS images. If the images show non-uniformities, the foams are not used for experiments. Figure 4 shows end-on images of two sample foams. The images show both a uniformly doped foam and a chunky foam where the Ti particles coagulate to the desired size.

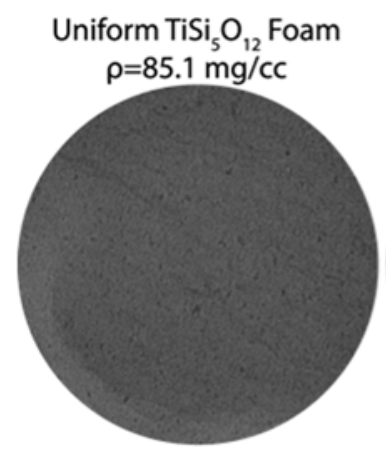

a.)

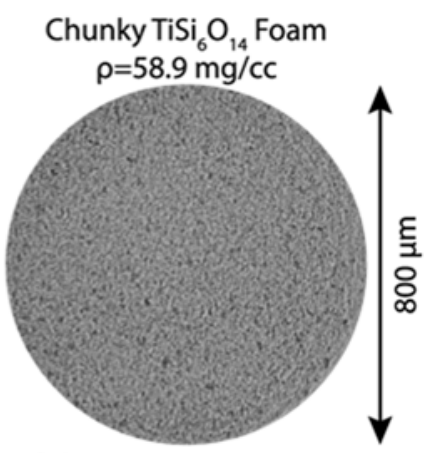

b.)
Figure 4. End-on images of two Ti-doped aerogel foams with (a) uniform and (b) chunky dopant profiles.

\section{Manufacturing and assembly}

Micro-machining processes have been developed to produce exacting fits between $\left(\mathrm{SiO}_{2}\right)$ aerogel cylinders and the doped aerogel inserts. Outer aerogel cylinders are machined to fit the final insertion diameter of the very thin-walled beryllium tubes. An intensive inspection process is required before machining, throughout machining and into the finished final assembly processes in order to produce these very delicate target assemblies.

Machining of the aerogel is done with Nakanishi Shika Kikai (NSK) live tooling spindles using a process called 'turn milling'. This process is useful because it eliminates any tool pressure, allowing us to take heavier roughing cuts without having the aerogel detach from the mandrel. This method also saves crucial machining time and allows more target hardware to be machined during a production run. The live tooling is set for 20,000 RPM, with the Hardinge spindle running at 12,000 RPM in the opposite direction. In the final procedure, a $12 \mu \mathrm{m}$ radius single crystal diamond tool is used for the final finishing of the O.D and the parting-off to finish length of the completed hardware. One live tooling mounted along the $z$-axis on the top plate is used for facing and roughing purposes. A second live tool spindle is set at $90^{\circ}$ along the $z$-axis for roughing out the backside. When finished, the part is done in one operation; there is no need for a second operation fixture, and hence, a time-saving process.

The first step in the assembly process is attaching the beryllium tube to the gold hohlraum. Manufacturing of the beryllium tubes uses the same technique as in Ref. [17]. The beryllium tube has three fiducial grooves machined on the outer surface with different spacing between the grooves meaning the tube has a direction. Before attachment of the Be cylinder the orientation is checked by examining the distance between the fiducials on the ends of the tube. Then the Be tube is attached to the hohlraum using the triple theta assembly station ${ }^{[18]}$. Before gluing, the orientation of the cylinder and the alignment of the tube with respect to the hohlraum is checked. In addition, the seating of the cylinder 


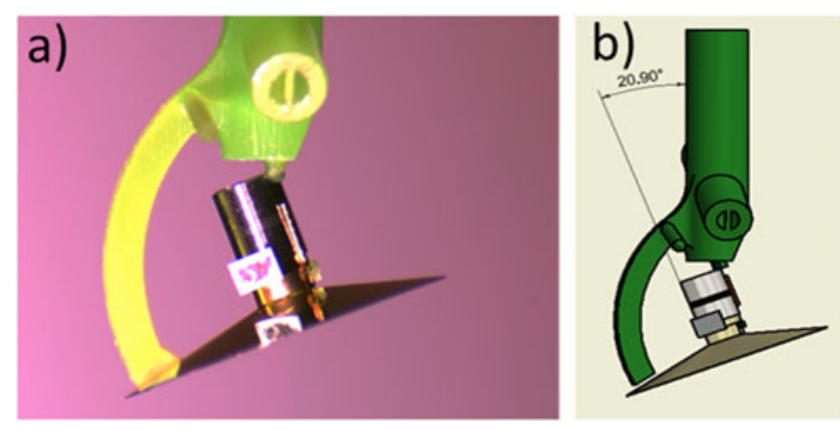

Figure 5. (a) Image of assembled target with CAD drawing showing the same view.

on the hohlraum holding ring is verified. The two pieces are glued using an ultraviolet adhesive.

The foam packages are prepared next. The larger undoped aerogel foam is placed on a vacuum tip with the bored out region facing upward using the triple theta assembly station $^{[18]}$. Special care must be taken when handling these foams as they are very delicate and difficult to see. The foam is leveled and centered. The Sc- or Ti-doped foam is picked up with a small vacuum tip using the 5th-axis arm. The foam is also leveled and centered. Leveling and centering the two foams is a critical step since damage to both foams could occur if they are not aligned during insertion. Once both foams are centered and leveled, the $\mathrm{Sc} / \mathrm{Ti}$ foam is slowly inserted into the $\mathrm{SiO}_{2}$ foam. While inserting the foam, a side camera and a front camera are used to watch the insertion process to ensure the smaller foam is completely pushed in and to verify there are no gaps before turning off the vacuum to release the doped aerogel.

Once the foams and the hohlraum with the Be tube are assembled, the foams are inserted into the beryllium cylinder. Again, this is done using the triple theta assembly station $^{[18]}$. The two parts are centered and leveled. The Be tube and hohlraum are lowered over the foam. After the foam is inserted, care is taken to ensure that there is no gap between the washer at the end of the half-hohlraum. Then small glue beads are placed at the top foam $/ \mathrm{Be}$ cylinder interface on opposite sides. A gold cone shield is added around the laser entrance hole of the hohlraum to complete the main target build.

The last step is mounting the main target to the stalk (Figure 5). The stalk consists of a rod with a rapid prototype 3D printed holder ${ }^{[19]}$. The rapid prototype holders are ordered from Fineline Proto Labs ${ }^{[20]}$. The microfine green material is an approved material at the Omega laser facilities and the NIF. Using the 3D printed holder simplifies attaching the main target to the stalk since the design allows the target to rest in the holder. The half-hohlraums are mounted to the prototype holders using the hohlraum mounting station (HMS). After mounting, the position on the holder is verified and recorded on the shadow graph before finally taking $\mathrm{Xradia}^{[8]}$ images to check for gaps, defects and additional measurements.

\section{Conclusions}

Techniques developed to construct targets for absorption spectroscopy with high-z dopants have been described. The key developments for these targets have been the ability to dope aerogel foams with nanoparticles and machine them such that a doped foam can be inserted into an undoped foam. This work has enabled absorption spectroscopy targets that can be driven on the NIF to higher material temperatures than previous experiments while measuring the supersonic heat wave driven by the hohlraum.

\section{Acknowledgment}

This work performed under the auspices of the U.S. Department of Energy by LANL under contract DE-AC5206NA25396.

\section{References}

1. D. Hoarty, L. Barringer, C. Vickers, O. Willi, and W. Nazarov, Phys. Rev. Lett. 82, 3070 (1999).

2. D. Hoarty, O. Willi, L. Barringer, C. Vickers, R. Watt, and W. Nazarov, Phys. Plasmas 6, 2171 (1999).

3. O. Willi, L. Barringer, C. Vickers, and D. Hoarty, Astrophys. J. Suppl. Ser. 127, 527 (2000).

4. J. A. Paisner, E. M. Campbell, and W. J. Hogan, Fusion Technol. 26, 755 (1994).

5. J. Fricke and T. Tillotson, Thin Solid Films 297, 212 (1997).

6. J. Hager, N. Lanier, and J. Kline, Rev. Sci. Instrum. (2016) submitted.

7. T. R. Boehly, R. S. Craxton, T. H. Hinterman, J. H. Kelly, T. J. Kessler, S. A. Kumpan, S. A. Letzring, R. L. McCrory, S. F. B. Morse, W. Seka, S. Skupsky, J. M. Soures, and C. P. Verdon, Rev. Sci. Instrum. 66, 508 (1995).

8. Zeiss, Carl-Zeiss-Strasse 22, 73447 Oberkochen, Germany, www.zeiss.de.

9. General Atomics, 3550 General Atomics Court, San Diego, CA $92121-1122$.

10. J. M. Taccetti, P. A. Keiter, N. Lanier, K. Mussack, K. Belle, and G. R. Magelssen, Rev. Sci. Instrum. 83, 023506 (2012).

11. C. E. Hamilton, D. Honnell, B. M. Patterson, D. W. Schmidt, and K. A. D. Obrey, Fusion Sci. Technol. 59, 194 (2011).

12. BOC science, 5-16 Ramsey Road, Shirley, NY 11967, USA, http://www.bocsci.com/.

13. Fisher Scientific, https://www.fishersci.com/us/en/home.html.

14. Sigma Aldrich Corp., St. Louis, MO, USA, https://www.sigm aaldrich.com/.

15. Goodfellows inc., 125 Hookstown Grade Road, Coraopolis, PA 15108-9302, USA, http://www.goodfellowusa.com/.

16. N.E. Lanier, C. Hamilton, and J. M. Taccetti, Rev. Sci. Instrum. 83, 10E521 (2012).

17. D. Capelli, D. W. Schmidt, T. Cardenas, G. Rivera, R. B. Randolph, F. Fierro, E. C. Merritt, K. A. Flippo, F. W. Doss, and J. L. Kline, Fusion Sci. Technol. 70, 316 (2016).

18. D. W. Schmidt, Fusion Sci. Technol. 55, 290 (2009).

19. T. Cardenas, D. W. Schmidt, and D. S. Peterson, Fusion Sci. Technol. 70, 288 (2016).

20. Proto Labs, 9310 Focal Point, Suite 100, Raleigh, NC 27617, https://www.protolabs.com/. 